\title{
Dissimilar evolutionary histories of two resistance gene families in the genus Solanum
}

\begin{tabular}{|r|l|}
\hline Journal: & Genome \\
\hline Manuscript ID & gen-2016-0101.R1 \\
\hline Manuscript Type: & Article \\
\hline Date Submitted by the Author: & 04-Jul-2016 \\
\hline Complete List of Authors: & $\begin{array}{l}\text { Segura, Diana; Universidad Nacional de Cuyo Facultad de Ciencias } \\
\text { Agrarias; IBAM - CONICET } \\
\text { Masuelli, Ricardo; Universidad Nacional de Cuyo Facultad de Ciencias } \\
\text { Agrarias; IBAM - CONICET } \\
\text { Sanchez-Puerta, María; Universidad Nacional de Cuyo Facultad de Ciencias } \\
\text { Agrarias; IBAM - CONICET }\end{array}$ \\
\hline Keyword: & resistance, <i>Solanum </i $>$, evolution, $<\mathrm{i}>$ Mi-1</i>, $<\mathrm{i}>$ Sw5</i> \\
\hline &
\end{tabular}

SCHOLARONE ${ }^{\text {It }}$

Manuscripts 
Dissimilar evolutionary histories of two resistance gene families in the genus Solanum

Diana María Seguraํㅜ, Ricardo Williams Masuelli¹ \& M. Virginia Sanchez-Puerta1,2*

1'IBAM, Universidad Nacional de Cuyo, CONICET, Facultad de Ciencias Agrarias, Almirante Brown 500, M5528AHB, Chacras de Coria, Argentina. ${ }^{2}$ Facultad de Ciencias Exactas y Naturales, Universidad Nacional de Cuyo, 5500, Mendoza, Argentina.

*Corresponding author: IBAM, Universidad Nacional de Cuyo, CONICET, Facultad de Ciencias Agrarias, Almirante Brown 500, M5528AHB, Chacras de Coria, Argentina Fax: 54-261-4960469

Ph: 54-261-4135000 ext1307

E-mail: mvsanchezpuerta@fca.uncu.edu.ar 
ABSTRACT

Genomic analyses have shown that most genes in eukaryotic lineages belong to families. Gene families vary in terms of number of members, nucleotide similarity, gene integrity, expression, and function. Often, the members of gene families are arranged in clusters, which contribute to maintaining similarity among gene copies and also to generate duplicates through replication errors. Gene families offer us an opportunity to examine the forces involved in the evolution of the genomes and to study recombination events and genomic rearrangements. In this work, we focused on the evolution of two plant resistance gene families, $S w 5$ and $M i-1$, and analyzed the completely sequenced nuclear genomes of potato and tomato. We first noticed that the potato genome carries larger resistance gene families than tomato, but all gene copies are pseudogenes. Second, phylogenetic analyses indicated that $S w 5$ and $M i-1$ gene families had dissimilar evolutionary histories. In contrast to $S w 5, M i-1$ homologues suffered repeated gene conversion events among the gene copies, particularly in the tomato genome.

Keywords: resistance, Solanum, evolution, Mi-1, Sw5. 


\section{INTRODUCTION}

New genes in a genome are commonly originated by duplication, chromosomal rearrangements, and subsequent divergence from pre-existing genes (Lawton-Rauh 2003). Gene duplication may occur by local or genome-wide events, such as polyploidization, and lead to the formation of a gene family. A gene family is a set of similar genes formed by duplication that generally share related biochemical functions. Most genes within a plant genome belong to gene families that originated as tandem duplicates, dispersed duplications, or genome-wide duplications, and are often arranged in clusters (Chen et al. 2007; Jia et al. 2015; Wu et al. 2014). A great number of gene families were described in plant genomes, including the phytochrome photoreceptor family in tomato (Alba 2000), the apyrase family in legumes that plays an important role in nutrition, photomorphogenesis, and nodulation (Cannon et al. 2003), and the calmodulin family in Solanaceae, which codes for different calcium sensor isoforms (Zhao et al. 2013). In addition, most resistance genes belong to gene families and are found in clusters (Hulbert et al. 2001; Andolfo et al. 2013). In some cases, gene family members are highly similar and produce large amounts of a single product, usually needed in a demanding metabolic process (Walsh et al. 2001). These gene families are subject to concerted evolution, maintaining highly similar copies by repeated gene conversion. In contrast, other gene families present dissimilar copies that recombine and exchange fragments, generating new allelic forms and increased variability (Ashfield et al. 2012). In addition, gene families whose members are arranged in clusters can suffer gene-size amplification by tandem duplications (due to replication slippage or unequal recombination), duplications of large regions (even the whole genome), transposition of DNA sequences, or retrotransposition of RNA transcripts (Thornton et al. 2000). The birth-and-death model of gene families implies that whereas some duplicated genes are maintained in the genome for a long time, others are deleted or inactivated through deleterious mutations (Nei and Rooney 2005). It has been suggested that disease resistance loci followed this model of evolution in plants (Michelmore and Meyers 1998; Nam et al. 2004). 
Their selective effects and population genetic parameters, such as effective population size, will determine the fate of these genomic changes.

In this work, we studied the evolution of two resistance gene families in the genus Solanum, family Solanaceae. We focused on resistance gene families because they show a complex evolution in previous studies of the family Solanaceae (Bakker et al. 2011; Lozano et al. 2012). The resistance gene family, $S w 5$, which was described in the wild tomato Solanum peruvianum (Spassova et al. 2001), is involved in the resistance to the Tomato Spotted Wilt Virus. In particular, the $S w 5-b$ gene copy confers resistance to this virus, and it has been mapped to the long arm of chromosome 9 in the tomato genome (Chagué et al. 1996; Stevens et al. 1992, 1995). The gene Mi-1.2 confers resistance to the potato aphid Macrosiphum euphorbiae, the whitefly Bemisia tabaci, and a group of root-knot nematodes of the genus Meloidogyne (Dropkin 1969; Nombela et al. 2003; Rossi et al. 2003; Jablonska et al. 2007). This gene belongs to the Mi-1 gene family, which forms a cluster of seven copies located in the short arm of chromosome 6 and two copies located in chromosome 5 of the Solanum lycopersicum nuclear genome (Seah et al. 2004; 2007). Approximately $45 \mathrm{Mi}-1$ homologues were identified in the genome of Solanum tuberosum diploid heterozygous line RH89-039-16; five times more than those in tomato (Sanchez-Puerta and Masuelli 2011). However, because the potato line analyzed was heterozygous, it was difficult to determine whether those homologues were actual genes or different alleles (Sanchez-Puerta and Masuelli 2011). The genomic arrangement, number of copies, integrity, and evolution of these gene families in solanaceous species remain unclear.

Recently, complete genomic sequences of three Solanaceae species were published: the double monoploid line S. tuberosum Group Phureja DM1-3 516R44 (The Potato Genome Sequencing Consortium 2011), S. lycopersicum cultivar 'Heinz 1706' (The Tomato Genome Consortium 2012), and Capsicum annuum cultivar CM334 (Kim et al. 2013). The availability of these genomes enabled us to study gene families in three important and closely related crops by comparative genomics. To contrast the evolution of the multigene families $S w 5$ and $M i-1$, we included three smaller resistance genes families (Hero, Hpa2, and Pto) and three single-copy 
genes (Waxy, Atg-1, Ufm-1) not related to pathogen resistance.

\section{MATERIALS AND METHODS}

\section{Nucleotide data}

The genomes of Solanum lycopersicum cultivar 'Heinz 1706' and a double monoploid of Solanum tuberosum Group Phureja DM1-3 516R44 were analyzed with Blast. Query sequences included the $S w 5$-b gene from S. lycopersicum cultivar Stevens (AY007366; $3740 \mathrm{bp}$ ), the Mi-1.2 gene from S. lycopersicum variety Motelle (AF091048, 3849 bp), Hero from S. lycopersicum (NM_001247066; 4280 bp), Gpa2 from S. tuberosum (AF195939; 2710 bp), Pto from S. peruvianum (DQ019220; 915 bp), Ufm-1 from Arabidopsis thaliana (NM_106420.4; 647 bp), Waxy from S. tuberosum (EU548081.1; 1320 bp), and Agt1 from A. thaliana (NM_126925.4; 1540 bp). All hits with coverage $>450 \mathrm{bp}$ and E-value $<1 \mathrm{e}^{-50}$, including full length and truncated copies, were extracted from the genomic sequences. Coding sequences from Capsicum annuum cultivar CM334 genome (Kim et al. 2014) were analyzed with BLAST to identify all homologues to the genes $S w 5-b$ and Mi-1.2. C. annuum is closely related to the genus Solanum and it can be helpful to analyze the evolutionary history of resistance gene families in potato and tomato. Chromosome maps were drawn with Vector NTI (Lu et al. 2005) and edited with Adobe Illustrator. The approximate location of centromeres in the potato and tomato chromosomes was estimated from Park et al. (2007) and SolGenomics Network, respectively.

\section{Phylogenetic analyses}

Sw5 and Mi-1 homologues identified in potato, tomato, and hot pepper were aligned individually using MacClade (Maddison and Maddison, 2000). Each nucleotide alignment was subjected to manual editing taking into account the encoded amino acid sequences to identify positional homology. Maximum likelihood analyses were performed with Garli 0.951 (Zwickl 2006) under the General Time Reversible model with parameters for invariant sites and gammadistributed rate heterogeneity ( 4 categories). One hundred bootstrap replicates were performed. 


\section{RESULTS}

Sw5 gene family is four times greater in potato than in tomato

BLAST searches of $S w 5-b$ against the nuclear genomes of $S$. lycopersicum and $S$. tuberosum yielded 9 (SlySw5h) and 36 (StbSw5h) homologues, respectively (Table S1). Only one homologue (SlySw5h3 from tomato) covered the entire gene length (3740 bp) and the rest were variable in size (due to indels), ranging from 490 to 4,572 bp (Table S1). Out of 45 Sw5 homologues identified in both Solanum species, 44 were classified as pseudogenes because they were truncated, contained premature stop codons, or had indels that led to frameshifts. The identity between potato homologues and the gene $S w 5-b$ was $73-94 \%$; and between tomato homologues and $S w 5-b$ was $77-97 \%$ (Table S1). We identified $26 S w 5$ homologues in Capsicum annuum, which were located in chromosomes 3, 7, and 10 (Table S3). The Sw5 tomato homologues were found in four chromosomes ( $\operatorname{chr} 1,6,9$ and 12), while those in the potato genome were identified in seven different chromosomes ( $\operatorname{chr} 1,5,6,7,8,9$ and 11) (Figure 1). There were several non-syntenic $S w 5$ homologues between potato and tomato genomes, such as those found in the potato chromosomes 1, 5, 6, 7, 8 and 11 and those in chromosomes 6 and 12 of tomato (Figure 1). Most Sw5 homologues were clustered in chromosomes 9 of both tomato and potato genomes. A particularly dense area encompassed 17 Sw5 homologues clustered within $\sim 360 \mathrm{~kb}$ of chromosome 9 of $S$. tuberosum.

\section{Mi-1 homologues were mainly pseudogenes and clustered in chromosome 6 of tomato and potato}

A BLAST search of Mi-1.2 homologues against the complete nuclear genomes of Solanum lycopersicum and S. tuberosum found 16 (SlyMih) and 38 (StbMih) gene copies, respectively, although most of them were not full-length (Table S2). The functional gene copy Mi-1.2 of $S$. lycopersicum has three exons, two of which (exons 2 and 3) are protein-coding exons. The first two exons and introns encompass 1,532 bp and were identified only in 5 homologues of the tomato genome under analysis (SlyMih10, SlyMih11, SlyMih12, SlyMih13, and SlyMih14), and in 8 of the 38 homologues in the potato genome (StbMih3, StbMih6, StbMih7, StbMih8, StbMih12, 
StbMih14, StbMih17, and StbMih18). Exon 3 sequences were recognized in all homologues of tomato and potato showing variable lengths (Table S2). Out of the 54 Mi-1 homologues identified in both genomes, 51 were classified as pseudogenes because they were truncated, contained premature stop codons, or had indels that led to frameshifts. Pseudogenes were variable in size, ranging from 717 to 5077 bp due to the occurrence of indels. The identity of each potato gene sequence to the tomato Mi-1.2 copy ranged between 76-92\%. The three fulllength Mi-1 copies (SlyMih11, SlyMih13, and SlyMih14) were found in the tomato genome and no complete genes were found in the potato genome. The Mi-1 gene family in Capsicum annuum had 71 members located in chromosomes 2, 5 and 6 (Table S3). Mi-1 homologues were found in three tomato chromosomes ( $\operatorname{chr} 5,6$ and 9), and five chromosomes of potato (chr 2, 4, 5, 6 and 9) (Figure 1). Tomato Mi-1 homologues present in chromosome 6 clustered in 2 loci, while those in potato clustered in five loci of the same chromosome (Figure 1). The three potato-specific clusters in chromosome 6, along with the homologues StbMih1 (in chromosome 2), StbMih2 (in chromosome 4) and several copies in chromosome 5 have no syntenic copies in tomato (Figure 1).

Comparison with other resistance gene families and single copy genes

Blast searches of additional resistance gene families, Hero, Gpa2, and Pto, against the nuclear genomes of potato and tomato also yielded a greater number of gene copies in $S$. tuberosum than in S. lycopersicum and those copies were distributed in a higher number of chromosomes of the potato genome (Table 1). In contrast, low-copy genes (Ufm-1, Waxy and Agt1) had only one copy of each gene in the nuclear genomes of potato and tomato and they were in syntenic locations (Table 1).

\section{Evolutionary analyses of Sw5 homologues and their genomic distribution}

The phylogenetic tree based on Sw5 sequences showed that tomato, potato, and hot pepper Sw5 homologues did not form species-specific monophyletic groups (Figure 2A). In most 
cases, homologues found in the same chromosomes in both potato and tomato did not group together in the tree. The distribution of the $S w 5$ in chromosomes 9 of potato and tomato was compared to their evolutionary relationships (Figure 2B). Along chromosome 9, tomato Sw5 homologues were found in three loci $\left(\mathrm{A}^{\prime}, \mathrm{B}^{\prime}\right.$ and $\left.\mathrm{C}^{\prime}\right)$, and potato homologues in six, where loci $\mathrm{D}, \mathrm{E}$ and $\mathrm{F}$ were not present in tomato. In addition, 17 potato homologues formed a large cluster (Figure 2B, cluster C). Based on the phylogenetic tree (Figure 2A), most of these clustered homologues were closely related to each other, except for StbSw5h8 and StbSw5h21. Finally, we observed a strongly supported sister relationship between the gene copy used as query for the Blast analyses $(S w 5 b)$ and the only putatively functional copy of $S w 5$ identified in this study, SlySw5h3 (Figure 2A).

\section{Evolutionary analyses of Mi-1 homologues}

A phylogenetic analysis of $M i-1$ homologues showed that potato and hot pepper sequences were more numerous and diverse than those from tomato (Figure 3A). All homologues from the genus Solanum formed a monophyletic clade divided in two main groups: i) one clade formed by all sequences from chromosome 6 of either tomato or potato, the SlyMih1 and SlyMih2 genes from tomato chromosome 5, and the genes StbMih2 and StbMih36 from potato chromosomes 4 and 5, respectively; and ii) the other clade included the majority of homologues located in chromosomes 2, 5, and 9 of either species (Figure 3A). All but one tomato homologue from chromosome 6 clustered in a single highly supported group (BS=100\%) embedded within a group of potato sequences from chromosome 6 . In contrast, tomato $\mathrm{Mi}-1$ homologues from chromosomes 5 and 9 were interspersed with potato homologues from chromosomes 2, 5, and 9. Individual tomato and potato sequences were not found as sister taxa, except for three pairs of homologues from different chromosomes (Figure 3A, brackets). In addition, we observed a strongly supported sister relationship between the gene copy used as query for the Blast analyses (Mi-1.2) and one of the functional copies of $M i-1$ identified in this study, SlyMih11 (Figure 2A). 
Comparing the physical location of Mi-1 homologues in chromosome 6 of both Solanum species, we observed that $M i-1$ copies formed clusters in two and five loci in tomato and potato, respectively (Figure 3B). Cluster C in potato was the most highly populated. All homologues found in the two tomato clusters, except for SlyMih16, formed a well-supported clade in the evolutionary tree, closely related to the potato homologues found in chromosome 6 (Figure 3A). Comparative analyses of the potato and tomato clusters revealed the presence of highly similar intergenic regions between clusters A and A' from potato and tomato, respectively, indicating they were homologous. However, gene copies found in the clusters A and A' did not group together in the phylogenetic tree (Figure 3A).

\section{DISCUSSION}

The availability of genomic sequences from S. tuberosum and S. lycopersicum allowed us to study the evolutionary history of resistance genes in two closely related species of the family Solanaceae. Most resistance genes are organized in tandem arrays, clusters, and super-clusters (Chen et al. 2007; Andolfo et al. 2013). In this study, tomato and potato sequences of two resistance gene families were often found in clusters, with exceptional members that were distantly located possibly due to duplications followed by translocations. Large resistance gene clusters provide a huge potential for the generation of sequence variation through recombination, facilitating the creation of new alleles or genes upon which pathogen selective pressures act. The investigation of the spatial arrangement of resistance clusters is useful in reconstructing the history of the chromosomal rearrangement that shaped the genome architecture of the ancestral species.

The comparative study we undertook indicated that all gene families analyzed were more numerous in S. tuberosum than in S. lycopersicum, even though their genomes are roughly equal in size (705 Mb and $759 \mathrm{Mb}$, respectively). This phenomenon may be due to successive duplications in the potato genome or repeated gene losses in tomato. In previous surveys, syntenic comparisons between the tomato and potato genomes indicated that tomato suffered 
the deletion of roughly one-third of the redundant proteome and transposable elements in euchromatic regions (The Tomato Genome Consortium 2012). This study also revealed that the tomato genome has approximately an equal number of resistance genes as $A$. thaliana, but fewer than grape, rice, and the potato genomes (The Tomato Genome Consortium 2012). Plant resistance gene family evolution has probably been influenced by polyploidization, genome size variation, natural and artificial selection including domestication breeding and cultivation, and gene family interactions (Jia et al. 2015; Wu et al. 2014; Zhong 2015). Given that the family Solanaceae evolved largely in the absence of polyploidization, except for the cultivated potato, but this event is insignificant in evolutionary times, any increase in the size of resistance gene families should be due to segmental duplications (Wu et al. 2010). Cross-hybridization experiments in Solanum showed that resistance genes have an ancient origin that predated speciation events in the Solanaceae (Quirin et al. 2012). After that, tomato and potato genomes suffered small and large rearrangements, along with duplications and deletions that obscured orthologous relationships because neither gene content nor order is strictly conserved (Andolfo et al. 2013).

The evolutionary histories of Sw5 and Mi-1 gene families were markedly different. Individual tomato $S w 5$ homologues maintained a close phylogenetic relationship with potato and hot pepper sequences. This suggests that several gene copies were present in the ancestor of these Solanaceae due to early segmental duplications. In contrast, sister relationships between tomato, potato and hot pepper $\mathrm{Mi}-1$ sequences were not observed. Instead, tomato homologues formed a highly supported monophyletic group, possible due to recurring gene conversion events within the tomato (Seah et al. 2007) and potato (Sanchez-Puerta and Masuelli 2011) genomes, respectively.

The tomato/potato Mi-1 homologues are distributed in less than perfectly matching positions across the two genomes, suggesting that conserved gene order on chromosomes is a mere vestige of common ancestry. SlyMih sequences of chromosome 6 are clustered in 2 loci, while StbMih sequences are found in 5 loci in the same chromosome. So, three potato clusters (C, 
D and E) of chromosome 6, StbMih1 from chromosome 2, StbMih2 from chromosome 4, and some genes from chromosome 5 in potato have no syntenic position in tomato. Novel clusters in potato may have been originated through translocation events. For example, a translocation of a segment of the ancestral cluster B of the potato chromosome 6 could have given rise to the first members of cluster C (Figure 3B). Evidence for this hypothesis includes the close relationship of StbMih33- StbMih26 and StbMih7-StbMih32 in the phylogenetic tree and the similarity of the intergenic sequences between these two pair of genes. Additional clusters of chromosome 6 of potato (clusters D and E) may have also originated by the duplication and translocation of an ancestral copy found in clusters C or A, respectively (Figure 3B). The great number of copies in cluster $\mathrm{C}$ was also puzzling. The increase in size of this cluster may have occurred by successive unequal crossing over events. The intimate phylogenetic relationships of some of the genes from this group (StbMih12-StbMih3-StbMih31, StbMih6-StbMih25-StbMih4, StbMih17-StbMih18, and StbMih30-StbMih8) were congruent with this hypothesis.

In contrast to the inferred evolution of the two resistance gene families, single copy genes did not suffer gene duplications in any of the genomes analyzed indicating that gene clusters are more prone to duplication and translocation through unequal crossing overs or errors in the replication or recombination processes (Krebs et al. 2013).

The distribution of homologous sequences in the genomes analyzed and the discovery of redundant genes provide insight into how novel pathogen-resistance genes can evolve via subsequent gene duplication events, unequal crossing over, translocation, and diversifying selection (Michelmore and Meyers 1998; Roth et al. 2007; Long et al. 2003). In conclusion, comparisons of gene arrangements between related plant genomes offer insight on their genome evolution. The complete sequencing of the tomato and potato genomes permitted an unprecedented view of Solanum resistance gene families, which may also impact in molecular breeding efforts.

\section{ACKNOWLEDGEMENTS}


This work was supported in part by SecTyP grant \#06/M053 from Universidad Nacional de Cuyo and grant PICT-277 from ANCyPT. RWM and MVSP are researchers of CONICET.

Conflict of interest: The authors declare that they have no conflict of interest.

\section{REFERENCES}

Alba, R., Kemelson, P., Cordonnier-Pratt, M. and Pratt, L. 2000. The phytochrome gene family in tomato and the rapid differential evolution of this family in angiosperms. Mol. Biol. Evol. 17(3):362-373.

Andolfo, G., Sanseverino, W., Rombauts, S., Van de Peer, Y., Bradeen, J.M., Carputo, D., Frusciante, L. and Ercolano, M.R. 2013. Overview of tomato (Solanum lycopersicum) candidate pathogen recognition genes reveals important Solanum R locus dynamics. New Phytologist 197: 223-237. Ashfield, T., Egan, A., Pfeil, B., Chen, N., Podicheti, R., Ratnaparkhe, M., Ameline-Torregrosa, C., Denny, R., Cannon, S., Doyle, J., Geffroy, V., Roe, B., Maroof, M., Young, N. and Innes, R. 2012. Evolution of a complex disease resistance gene cluster in diploid Phaseolus and tetraploid Glycine. Plant Physiology 159:336-354

Bakker, E., Borm, T., Prins, P., van der Vossen, E., Uenk, G., Arens, M., de Boer, J., van Eck, H., Muskens, M., Vossen, J., van der Linden, G., van Ham, R., Klein-Lankhorst, R., Visser, R., Smant, G., Bakker, J. and Goverse, A. 2011. A genome-wide genetic map of NB-LRR disease resistance loci in potato. Theor. Appl. Genet. 123: 493-508.

Cannon, S. B., McCombie, W.R., Sato, S., Tabata, S., Denny, R., Palmer, L., Katari, M., Young, N.D. and Stacey, G. 2003. Evolution and microsynteny of the apyrase gene family in three legume genomes. Mol. Gen. Genomics 270: 347-361

Chagué, V., Mercier, J.C., Guénard, M., de Courcel, A. and Vedel, F. 1996. Identification and mapping on chromosome 9 of RAPD markers linked to $S w-5$ in tomato by bulked segregant analysis. Theor. Appl. Genet. 92: 1045-1051.

Chen, G., Pan, D., Zhou, Y., Lin, S. and Ke, X. 2007. Diversity and evolutionary relationship of 
nucleotide binding site-encoding disease-resistance gene analogues in sweet potato (Ipomea batatas Lam.). J. Biosci 32(4), 713-721.

Dropkin, V. 1969. Cellular responses of plants to nematode infections. Annu Rev Phytopathol 7: 101-122.

Hulbert, S., Webb, C., Smith, S. and Sun, Q. 2001. Resistance Gene Complexes: Evolution and Utilization. Annual Review of Phytopathology 39:285-312.

Jablonska, B., Ammiraju, J., Bhattarai, K., Mantelin, S., Martinez de Ilarduya, O., Roberts, P. and Kaloshian, I. 2007. The Mi-9 gene from Solanum arcanum conferring heat-stable resistance to root-knot nematodes is a homolog of Mi-1. Plant Physiol 143:1044-1054.

Jia YX, Yuan Y, Zhang Y, Yang S, Zhang X. 2015. Extreme expansion of NBS-encoding genes in rosaceae. BMC Genetics 16(1), 1.

Kim, S., Park, M., Yeom, S., Kim, Y., Lee, J. et al. 2014. Genome sequence of the hot pepper provides insights into the evolution of pungency in Capsicum species. Nature 46:270-279.

Krebs, J., Lewin, B., Goldstein, E.S. and Kilpatrick 2013. Lewin's essential genes. Jones \& Bartlett Publishers, pp. 847.

Lawton-Rauh, A. 2003. Evolutionary dynamics of duplicated genes in plants. Molecular Phylogenetic and Evolution 29 396-409.

Long, M., Beltrán, E., Thornton, K. and Wang, W. 2003. The origin of new genes: glimpses from the young and old. Nature Reviews vol. 4 865-865.

Lozano, R., Ponce, O., Ramirez, M., Mostajo, N. and Orjeda, G. 2012. Genome-wide identification and mapping of NBS encoding resistance genes in Solanum tuberosum Group Phureja. Plos One 7(4): e34775.

Lu, G. and Moriyama, E. N. 2005. Vector NTI, a balanced all-in-one sequence analysis suite. Briefings in Bioinformatics, Vol 5, No 4 578-588.

Maddison, W. and Maddison, P. 2000. MacClade version 4: analysis of phylogeny and character evolution. Sinauer Associates, Sunderland.

Michelmore, R.W. and Meyers, B.C. 1998. Clusters of resistance genes in plants evolve by 
divergent selection and a birth-and-death process. Genome Res. 8:1323-1344.

Nam, J., de Pamphilis, C.W., Ma, H., Nei, M. 2004. Type I MADS-box genes have experienced faster birth-and-death evolution than type II MADS-box genes in angiosperms. Proc. Natl. Acad. Sci. USA 101:1910-1915.

Nei, M., Rooney, A.P. 2005. Concerted and birth-and-death evolution of multigene families. Annual Review Genet. 39:121-152.

Nombela, G., Williamson, V., Muniz, M. 2003. The root-knot nematode resistance gene Mi-1.2 of tomato is responsible for resistance against the whitefly Bemisia tabaci. Mol. Plant Microbe Interact. 16:645-649.

Park, T., Kim, J., Hutten, R., van Eck, Jacobsen, E. and Visser, R. 2007. Genetic positioning of centromeres using half-tetrad analysis in a $4 \mathrm{x}-2 \mathrm{x}$ cross population of potato. Genetic Society of America 176: 85-94.

Philippe, H., Snell, E., Bapteste, E., Lopez, P., Holland, P. and Casane, D. 2004. Phylogenomics of eukaryotes: impact of missing data on large alignments. Mol. Biol. Evol. 21:1740-1752.

Quirin, E., Mann, H., Meyer, R., Traini, A., Chiusano, M. L., Litt, A. and Bradeen, J. 2012. Evolutionary meta-analysis of Solanaceous resistance gene and Solanum resistance gene analog sequences and practical framework for cross-species comparisons. Mol. Plant-Microbe Interact Vol. 25, No. 5, 603-612.

Rossi, M., Goggin, F., Milligan, S., Kaloshian, I., Ullman, D. and Williamson. V. 2003. The nematode resistance gene $M i$ of tomato confers resistance against the potato aphid. Proc Natl Acad Sci USA 95:9750-9754.

Roth, C., Rastogi, S., Arvestad, L., Dittmar, K., Light, S., Ekman, D. and Liberles, D. 2007. Evolution after gene duplication: models, mechanisms, sequences, systems and organisms. J. Exp. Zool. (Mol. Dev. Evol.) 308B:58- 73.

Sanchez-Puerta, M. V. and Masuelli, R. W. 2011. Evolution of nematode-resistant Mi-1 gene homologues in three species of Solanum. Mol Genet Genomics 285:207-218.

Sasser, J. and Carter, C. 1985. An advance treatise on Meloidogyne. NC State Univ Graph, Raleigh

https://mc06.manuscriptcentral.com/genome-pubs 
Seah, S., Yaghoobi, J., Rossi, M., Gleason, C. A. and Williamson, V. M. 2004. The nematoderesistance gene, Mi-1, is associated with an inverted chromosomal segment in susceptible compared to resistant tomato. Theor Appl Genet 108:1635-1642

Seah, S., Telleen, A., Williamson, V. 2007. Introgressed and endogenous Mi-1 gene clusters in tomato differ by complex rearrangements in flanking sequences and show sequence exchange and diversifying selection among homologues. Theor. Appl. Genet. 114:1289-1302 Spassova, M., Prins, T., Folkertsma, R., Klein-Lankhorst, R., Hille, J., Goldbach, R. and Prins, M. 2001. The tomato gene Sw5 is a member of the coiled coil, nucleotide binding, leucine-rich repeat class of plant resistance genes and confers resistance to TSWV in tobacco. Molecular Breeding 7: 151-161.

Stevens, M. R., Scott, S. J. and Gergerich, R. C. 1992. Inheritance of a gene for resistance to tomato spotted wilt virus (TSWV) from Lycopersicon peruvianum Mill. Euphytica 59: 9-17.

Stevens, M. R., Lamb, E. M. and Rhoads, D. D. 1995. Mapping the $S w-5$ locus for tomato spotted wilt virus resistance in tomatoes using RAPD and RFLP analyses. Theor. Appl. Genet. 90: 451456.

Thornton, J. and DeSalle, R. 2000. Gene family evolution and homology: genomics meets phylogenetics. Annu. Rev. Genomics Hum. Genet. 01:41-73.

The Potato Genome Sequencing Consortium 2011. Genome sequence and analysis of the tuber crop potato. Nature 475: 189-195.

The Tomato Genome Consortium 2012. The tomato genome sequence provides insights into fleshy fruit evolution. Nature 485: 635-641

Wiens, J. 2006. Missing data and the design of phylogenetic analyses. J Biomed Inform 39:34-42

Walsh, B. and Stephan, W. 2001. Multigene families: Evolution. Encyclopedia of Life Sciences

Wu, F. and Tanksley, S. D. 2010. Chromosomal evolution in the plant family Solanaceae. BMC Genomics 11:182. 
Wu P, Shao ZQ, Wu XZ, Wang Q, Wang B, Chen JQ, Hang YY, Xue JY. 2014. Loss/retention and evolution of NBS encoding genes upon whole genome triplication of Brassica rapa. Gene 540 (1), $54-61$.

Zhao, Y., Liu, W., Xu, Y., Cao, J., Braam, J. and Cai, X. 2013. Genome-wide identification and functional analyses of calmodulin genes in Solanaceous species. BMC Plant Biology 13:70.

Zhong Y, Yin H, Sargent DJ, Malnoy M, Cheng ZM. 2015. Species-specific duplications driving the recent expansion of NBS-LRR genes in five Rosaceae species. BMC Genomics 16(1), 1.

Zwickl, D. J. 2006. Genetic algorithm approaches for the phylogenetic analysis of large biological sequence datasets under the maximum likelihood criterion. University of Texas, Austin. 


\section{TABLES}

Table 1. Number of gene copies in the tomato and potato genomes

\begin{tabular}{|c|c|c|c|}
\hline Gene name & $\begin{array}{l}\text { Chromosome } \\
\text { number }^{\mathrm{a}}\end{array}$ & $\begin{array}{l}\text { Number of gene } \\
\text { copies in } S \text {. } \\
\text { lycopersicum }\end{array}$ & $\begin{array}{l}\text { Number of } \\
\text { gene copies in } \\
\text { S. tuberosum }\end{array}$ \\
\hline \multirow[t]{5}{*}{$M i-1.2(3732 \mathrm{bp})$} & 2 & 0 & 1 \\
\hline & 4 & 0 & 1 \\
\hline & 5 & 8 & 4 \\
\hline & 6 & 7 & 31 \\
\hline & 9 & 1 & 1 \\
\hline \multirow[t]{7}{*}{$S w 5-b(3741 \mathrm{bp})$} & 1 & 1 & 5 \\
\hline & 5 & 0 & 2 \\
\hline & 6 & 1 & 3 \\
\hline & 7 & $x$ & 1 \\
\hline & 8 & 0 & 1 \\
\hline & 9 & 6 & 23 \\
\hline & 12 & 1 & 0 \\
\hline \multirow[t]{4}{*}{ Hero (4280 bp) } & 3 & 1 & 1 \\
\hline & 4 & 8 & 11 \\
\hline & 6 & 1 & 1 \\
\hline & 9 & 1 & 1 \\
\hline \multirow[t]{2}{*}{ Gpa2 (3208 bp) } & 10 & 0 & 1 \\
\hline & 12 & 3 & 15 \\
\hline \multirow[t]{3}{*}{ Pto (915 bp) } & 3 & 0 & 1 \\
\hline & 5 & 5 & 6 \\
\hline & 6 & 1 & 1 \\
\hline
\end{tabular}




\begin{tabular}{|l|c|c|c|} 
& 11 & 1 & 1 \\
\hline Waxy $(1320 \mathrm{bp})$ & 8 & 1 & 1 \\
\hline Agt-1 (1090 bp) & 12 & 1 & 1 \\
\hline Ufm-1 (283 bp) & 12 & 1 & 1 \\
\hline
\end{tabular}

${ }^{a}$ Chromosomes that are not listed, do not contain gene copies for the corresponding gene family. 
Figure 1. Physical location of $M i-1$ and $S w 5$ homologues of tomato and potato. Tomato and potato chromosomes are represented in white (T) and grey (P), respectively. Sw5 and Mi-1 homologues are indicated on the right and left of each chromosome, respectively. Missing chromosomes do not contain any Mi-1 or Sw5 homologues. The approximate location of centromeres in the potato and tomato chromosomes was estimated from Park et al. (2007) and SolGenomics Network, respectively. 
Figure 2: Analyses of $\boldsymbol{S w 5}$ homologues. A. Maximum Likelihood phylogenetic tree of 71 sequences from Capsicum annuum (CA), S. lycopersicum (SlySw5h) and S. tuberosum (StSw5h) based on 3,741 bp. Numbers on branches correspond to bootstrap support values $>50 \%$ from 100 bootstrap replicates. The chromosome location $(\mathrm{Cn})$ is shown next to the gene name. $\mathbf{B}$. Graphical representation of Sw5 homologues located in chromosome 9 of S. lycopersicum (above) and S. tuberosum (below). Well-supported evolutionary relationships are indicated by solid lines. Arrows with numbers indicate $S w 5$ homologues and letters represent different loci. 
Figure 3: Analyses of Mi-1 homologues. A. Maximum Likelihood phylogenetic tree of 126 sequences from Capsicum annuum (CA), S. lycopersicum (SlyMih) and S. tuberosum (StbMih) based on 3,876 bp. Numbers on branches correspond to bootstrap support values $>50 \%$ from 100 bootstrap replicates. The chromosome location $(\mathrm{Cn})$ is shown next to the gene name. $\mathbf{B}$. Graphical representation of Mi-1 homologues from chromosome 6 from S. lycopersicum (above) and S. tuberosum (below). Well-supported evolutionary relationships are indicated by solid lines. Arrows with numbers indicate Mi-1 homologues and letters represent different loci. 

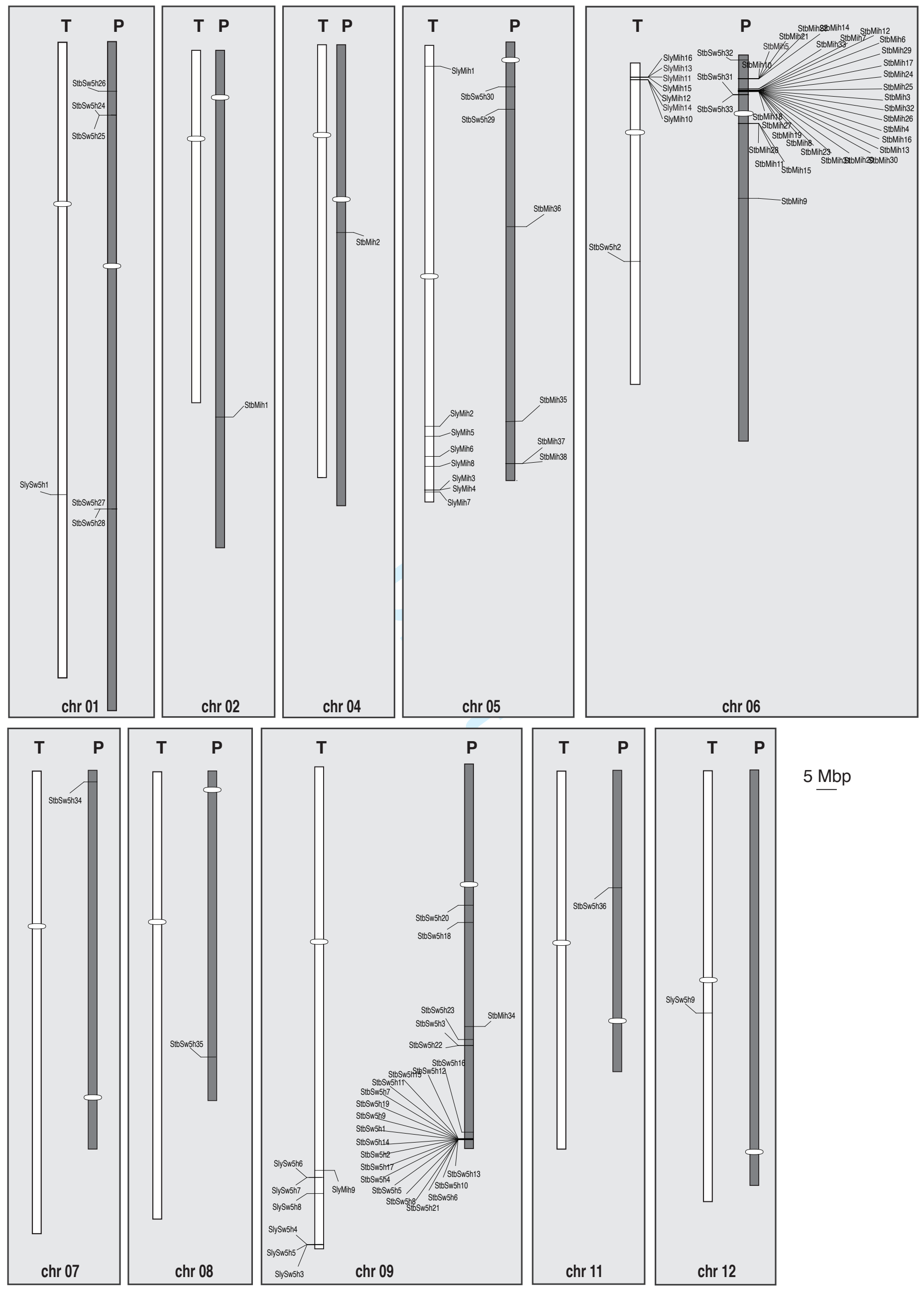


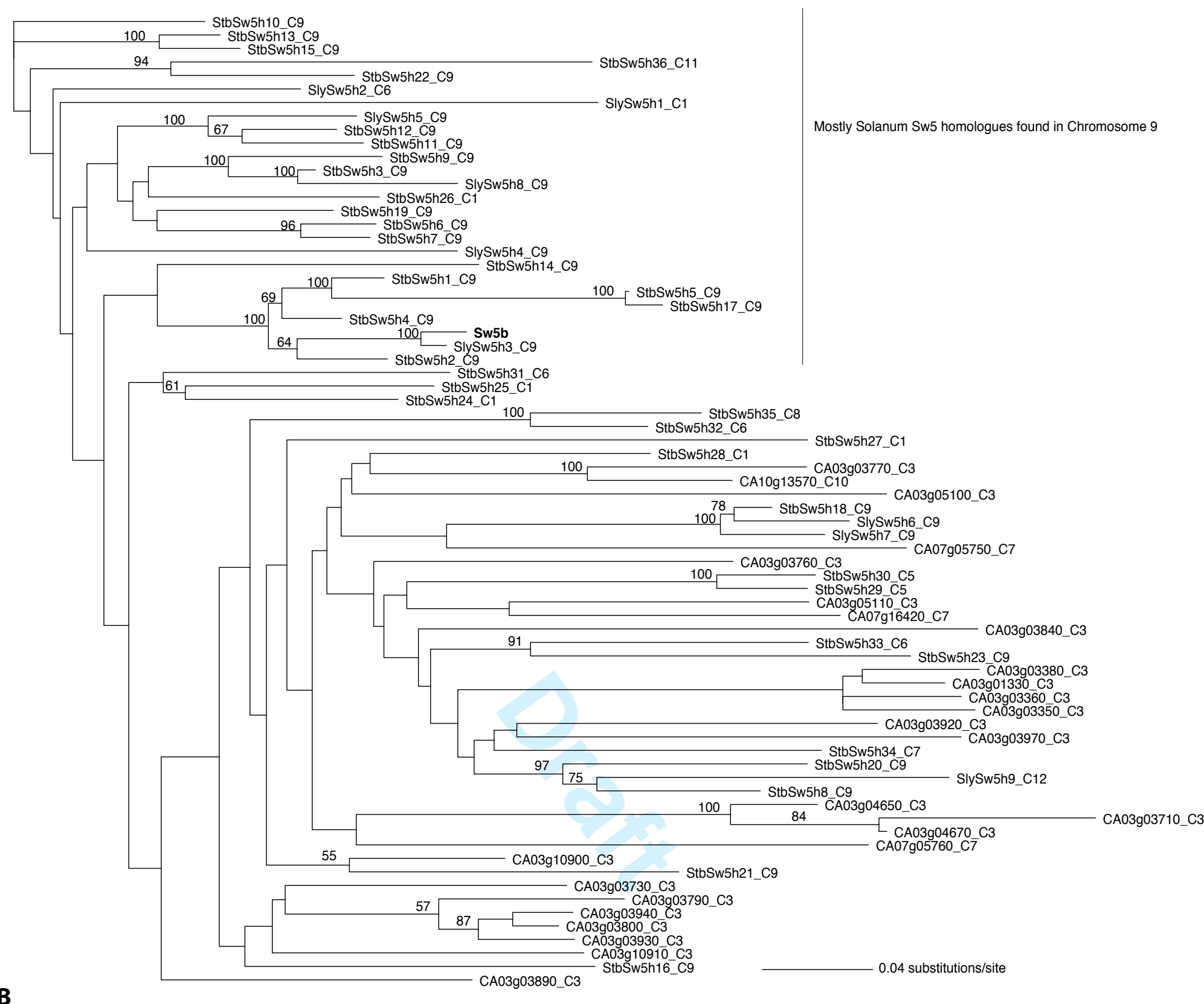

B

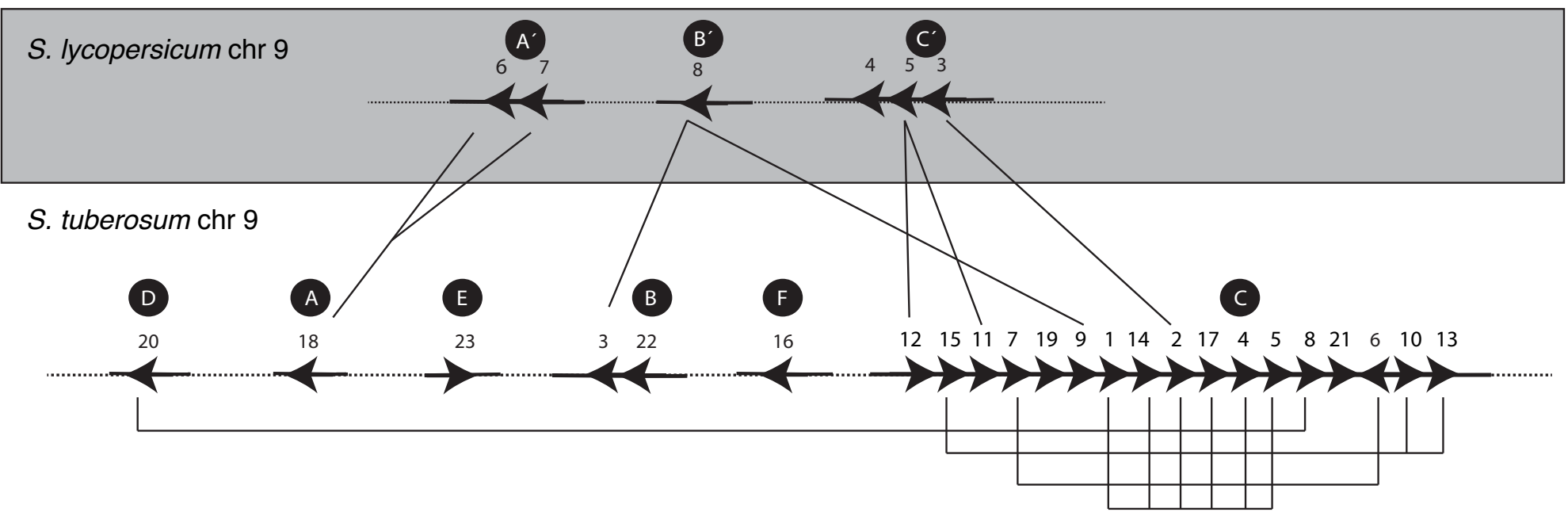


A

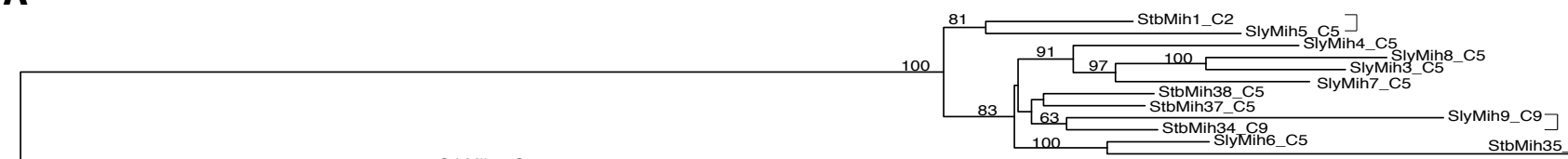
StbMin35_C5
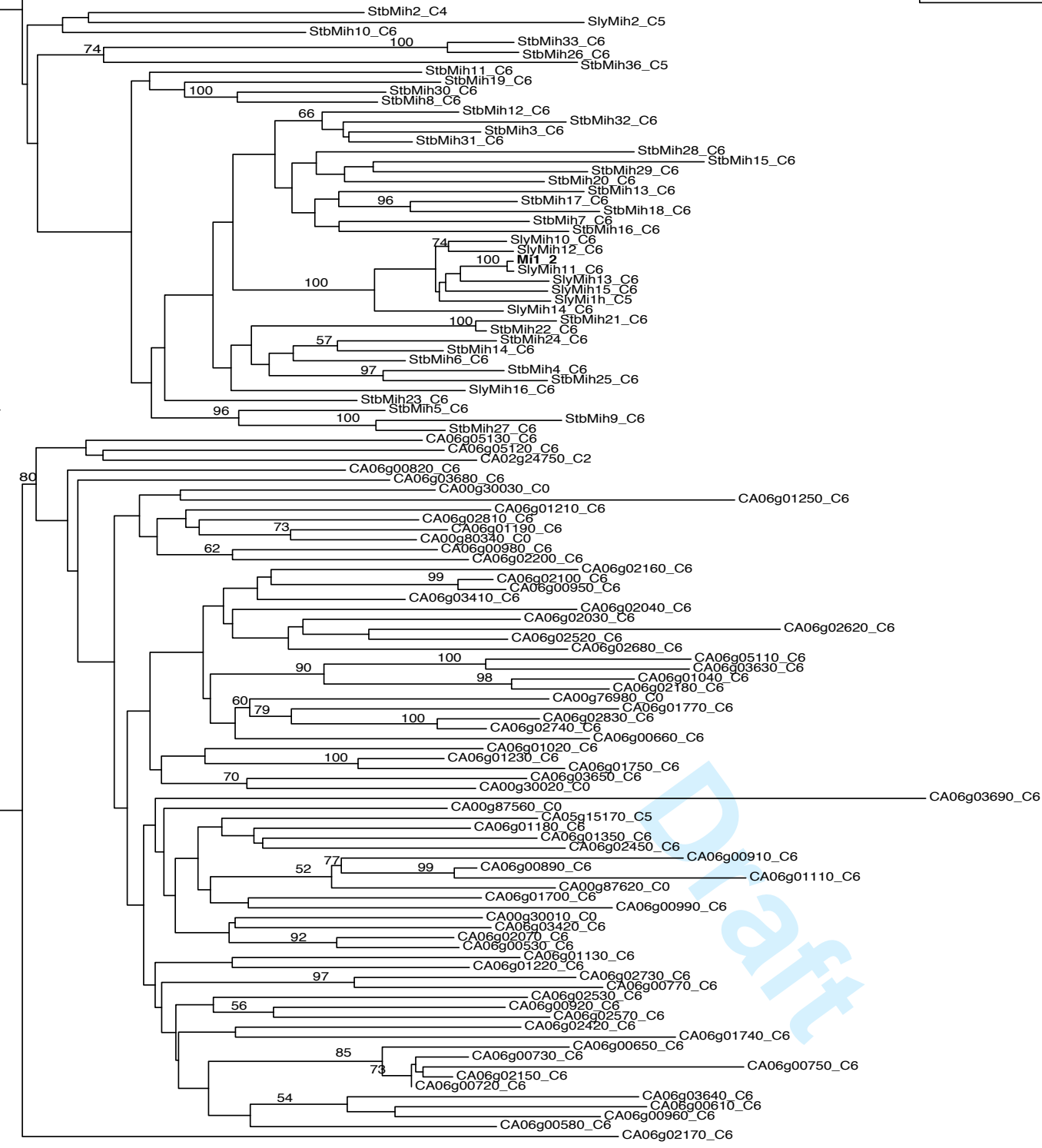

Capsicum annuum Mi-1 homologues

Solanum

Mi-1

homologues

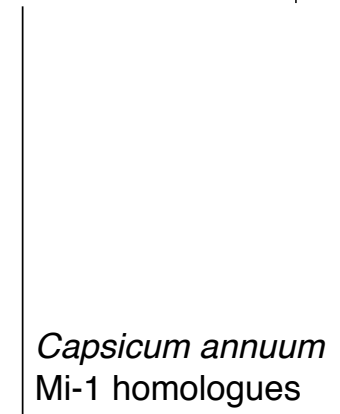

B

S. lycopersicum chr 6
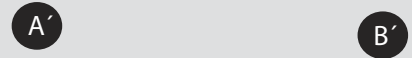

2,3 Mb

$2,7 \mathrm{Mb}$

$1613 \quad 11 \quad 15$

121410

HH+

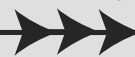

S. tuberosum chr 6

A B

2,7 Mb

$\begin{array}{lllll}10 & 5 & 21 & 22 & 14\end{array}$

HHT

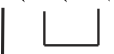

337

$4,5 \mathrm{Mb}$
C

$\begin{array}{llllllllllllllllllll}12 & 6 & 29 & 17 & 24 & 25 & 3 & 32 & 26 & 4 & 16 & 13 & 30 & 20 & 31 & 23 & 8 & 19 & 27 & 18\end{array}$

$\rightarrow$
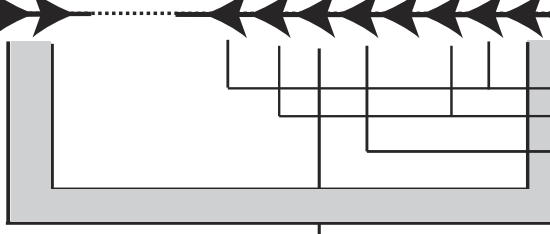
Table S1. Sw5 homologues in S. lycopersicum and S. tuberosum genomes.

\begin{tabular}{|c|c|c|c|c|c|c|}
\hline Gene Id & Species & Chromosome & Location & E-value & Pseudogene? & Length (bp) \\
\hline SlySw5h1 & S. lycopersicum & 1 & $64132079-64132569$ & $3 e-116$ & $\mathrm{y}$ & 490 \\
\hline SlySw5h2 & S. lycopersicum & 6 & $28770097-28769325$ & 0 & $\mathrm{y}$ & 772 \\
\hline SlySw5h3 & S. lycopersicum & 9 & $67305412-67301675$ & 0 & $\mathrm{n}$ & 3740 \\
\hline SlySw5h4 & S. lycopersicum & 9 & $67264887-67268567$ & 0 & $\mathrm{y}$ & 3680 \\
\hline SlySw5h5 & S. lycopersicum & 9 & $67271867-67274964$ & 0 & $\mathrm{y}$ & 3099 \\
\hline SlySw5h6 & S. lycopersicum & 9 & $57623293-57624987$ & 0 & $\mathrm{y}$ & 1694 \\
\hline SlySw5h7 & S. lycopersicum & 9 & $57632879-57633525$ & $2 \mathrm{e}-126$ & $\mathrm{y}$ & 646 \\
\hline SlySw5h8 & S. lycopersicum & 9 & $60416974-60416289$ & $4 e-59$ & $\mathrm{y}$ & 685 \\
\hline SlySw5h9 & S. lycopersicum & 12 & $36849257-36850871$ & 0 & $\mathrm{y}$ & 1614 \\
\hline StbSw5h1 & S. tuberosum & 9 & $52463812-52467551$ & 0 & $\mathrm{y}$ & 3739 \\
\hline StbSw5h2 & S. tuberosum & 9 & $52476100-52479765$ & 0 & $\mathrm{y}$ & 3665 \\
\hline StbSw5h3 & S. tuberosum & 9 & $39437902-39434178$ & 0 & $\mathrm{y}$ & 3724 \\
\hline StbSw5h4 & S. tuberosum & 9 & $52503565-52505756$ & 0 & $\mathrm{y}$ & 2191 \\
\hline StbSw5h5 & S. tuberosum & 9 & $52514572-52517091$ & 0 & $\mathrm{y}$ & 2519 \\
\hline StbSw5h6 & S. tuberosum & 9 & $52562918-52559071$ & 0 & $\mathrm{y}$ & 3847 \\
\hline StbSw5h7 & S. tuberosum & 9 & $52303902-52307768$ & 0 & $\mathrm{y}$ & 3866 \\
\hline StbSw5h8 & S. tuberosum & 9 & $52523818-52527160$ & 0 & $\mathrm{y}$ & 3342 \\
\hline StbSw5h9 & S. tuberosum & 9 & $52327226-52328474$ & 0 & $\mathrm{y}$ & 1248 \\
\hline StbSw5h10 & S. tuberosum & 9 & $52588516-52584861$ & 0 & $\mathrm{y}$ & 3655 \\
\hline StbSw5h11 & S. tuberosum & 9 & $52277856-52281626$ & 0 & $\mathrm{y}$ & 3770 \\
\hline StbSw5h12 & S. tuberosum & 9 & $52258466-52262124$ & 0 & $\mathrm{y}$ & 3658 \\
\hline StbSw5h13 & S. tuberosum & 9 & $52618296-52614558$ & 0 & $\mathrm{y}$ & 3738 \\
\hline StbSw5h14 & S. tuberosum & 9 & $52469931-52472995$ & 0 & $\mathrm{y}$ & 3064 \\
\hline StbSw5h15 & S. tuberosum & 9 & $52272853-52275919$ & 0 & $\mathrm{y}$ & 3066 \\
\hline StbSw5h16 & S. tuberosum & 9 & $51739970-51736787$ & 0 & $\mathrm{y}$ & 3183 \\
\hline StbSw5h17 & S. tuberosum & 9 & $52491452-52493110$ & 0 & $\mathrm{y}$ & 1658 \\
\hline StbSw5h18 & S. tuberosum & 9 & $22298490-22295479$ & 0 & $\mathrm{y}$ & 3011 \\
\hline StbSw5h19 & S. tuberosum & 9 & $52315099-52317260$ & 0 & $\mathrm{y}$ & 2161 \\
\hline StbSw5h20 & S. tuberosum & 9 & $19744467-19741900$ & 0 & $\mathrm{y}$ & 2567 \\
\hline StbSw5h21 & S. tuberosum & 9 & $52527371-52528596$ & 0 & $\mathrm{y}$ & 1225 \\
\hline StBSw5h22 & S. tuberosum & 9 & $39440348-39439645$ & $2 \mathrm{e}-165$ & $\mathrm{y}$ & 703 \\
\hline StbSw5h23 & S. tuberosum & 9 & $38423841-38424896$ & $8 e-85$ & $\mathrm{y}$ & 1055 \\
\hline StbSw5h24 & S. tuberosum & 1 & $10828634-10833206$ & 0 & $\mathrm{y}$ & 4572 \\
\hline StbSw5h25 & S. tuberosum & 1 & $10855462-10858705$ & 0 & $\mathrm{y}$ & 3243 \\
\hline StbSw5h26 & S. tuberosum & 1 & $6964707-6962519$ & 0 & $\mathrm{y}$ & 2188 \\
\hline StbSw5h27 & S. tuberosum & 1 & $66440727-66443772$ & 0 & $\mathrm{y}$ & 3045 \\
\hline StbSw5h28 & S. tuberosum & 1 & $66711701-66713709$ & 0 & $\mathrm{y}$ & 2008 \\
\hline StbSw5h29 & S. tuberosum & 5 & $9511029-9507974$ & 0 & $\mathrm{y}$ & 3055 \\
\hline StbSw5h30 & S. tuberosum & 5 & $6557047-6560112$ & 0 & $\mathrm{y}$ & 3065 \\
\hline StbSw5h31 & S. tuberosum & 6 & $5921825-5925565$ & 0 & $\mathrm{y}$ & 3740 \\
\hline StbSw5h32 & S. tuberosum & 6 & 738971-735677 & 0 & $\mathrm{y}$ & 3294 \\
\hline StbSw5h33 & S. tuberosum & 6 & $5956379-5957030$ & $2 e-85$ & $\mathrm{y}$ & 651 \\
\hline
\end{tabular}




\begin{tabular}{|l|c|c|c|c|c|c|}
\hline StbSw5h34 & S. tuberosum & 7 & $1352678-1350999$ & 0 & $\mathrm{y}$ & 1679 \\
\hline StbSw5h35 & S. tuberosum & 8 & $40493685-40492553$ & 0 & $\mathrm{y}$ & 1132 \\
\hline StbSw5h36 & S. tuberosum & 11 & $16500316-16503430$ & 0 & $\mathrm{y}$ & 3115 \\
\hline
\end{tabular}

${ }^{\mathrm{a}}$ The $\%$ identity was calculated by comparing to $S w 5-b$ gene. 


\begin{tabular}{|c|}
\hline \% Identity $^{\mathrm{a}}$ \\
\hline 83 \\
\hline 86 \\
\hline 97 \\
\hline 82 \\
\hline 84 \\
\hline 77 \\
\hline 80 \\
\hline 86 \\
\hline 81 \\
\hline 90 \\
\hline 92 \\
\hline 84 \\
\hline 94 \\
\hline 89 \\
\hline 85 \\
\hline 87 \\
\hline 80 \\
\hline 89 \\
\hline 84 \\
\hline 83 \\
\hline 84 \\
\hline 83 \\
\hline 84 \\
\hline 83 \\
\hline 80 \\
\hline 88 \\
\hline 79 \\
\hline 83 \\
\hline 80 \\
\hline 79 \\
\hline 82 \\
\hline 73 \\
\hline 84 \\
\hline 83 \\
\hline 84 \\
\hline 78 \\
\hline 81 \\
\hline 78 \\
\hline 78 \\
\hline 82 \\
\hline 80 \\
\hline 77 \\
\hline
\end{tabular}




\begin{tabular}{|l|}
\hline 80 \\
\hline 80 \\
\hline 84 \\
\hline
\end{tabular}


Table S2: Mi-1 homologues in S. lycopersicum and S. tuberosum genomes.

\begin{tabular}{|c|c|c|c|c|c|c|}
\hline Gene Id & Species & Chromosome & E-value & pseudogene? & Exon1+Intron1 & Exon2+Intron2 \\
\hline SlyMih1 & S. lycopersicum & 5 & 0 & $y$ & $\mathrm{n}$ & $\mathrm{n}$ \\
\hline SlyMih2 & S. lycopersicum & 5 & 0 & y & $\mathrm{n}$ & $\mathrm{n}$ \\
\hline SlyMih3 & S. lycopersicum & 5 & 0 & $y$ & $\mathrm{n}$ & $\mathrm{n}$ \\
\hline SlyMih4 & S. lycopersicum & 5 & 0 & y & $\mathrm{n}$ & $\mathrm{n}$ \\
\hline SlyMih5 & S. lycopersicum & 5 & 0 & $y$ & $\mathrm{n}$ & $\mathrm{n}$ \\
\hline SlyMih6 & S. lycopersicum & 5 & 0 & $y$ & $\mathrm{n}$ & $\mathrm{n}$ \\
\hline SlyMih7 & S. lycopersicum & 5 & 0 & $y$ & $\mathrm{n}$ & $\mathrm{n}$ \\
\hline SlyMih8 & S. lycopersicum & 5 & 0 & $y$ & $\mathrm{n}$ & $\mathrm{n}$ \\
\hline SlyMih9 & S. lycopersicum & 9 & 3 e-60 & y & $\mathrm{n}$ & $\mathrm{n}$ \\
\hline SlyMih10 & S. lycopersicum & 6 & 0 & $\mathrm{y}$ & $\mathrm{y}$ & $\mathrm{y}$ \\
\hline SlyMih11 & S. lycopersicum & 6 & 0 & $\mathrm{n}$ & y & $y$ \\
\hline SlyMih12 & S. lycopersicum & 6 & 0 & $y$ & $\mathrm{y}$ & y \\
\hline SlyMih13 & S. lycopersicum & 6 & 0 & $\mathrm{n}$ & $y$ & $y$ \\
\hline SlyMih14 & S. lycopersicum & 6 & 0 & $\mathrm{n}$ & y & y \\
\hline SlyMih15 & S. lycopersicum & 6 & 0 & $y$ & $\mathrm{n}$ & $\mathrm{n}$ \\
\hline SlyMih16 & S. lycopersicum & 6 & 0 & $y$ & $\mathrm{n}$ & $\mathrm{n}$ \\
\hline StbMih1 & S. tuberosum & 2 & 0 & $\mathrm{y}$ & $\mathrm{n}$ & $\mathrm{n}$ \\
\hline StbMih2 & S. tuberosum & 4 & 0 & $y$ & $\mathrm{n}$ & $\mathrm{n}$ \\
\hline StbMih3 & S. tuberosum & 6 & 0 & $y$ & $y$ & $y$ \\
\hline StbMih4 & S. tuberosum & 6 & 0 & $y$ & y & $\mathrm{n}$ \\
\hline StbMih5 & S. tuberosum & 6 & 0 & $y$ & $\mathrm{n}$ & $\mathrm{n}$ \\
\hline StbMih6 & S. tuberosum & 6 & 0 & $y$ & $\mathrm{y}$ & $\mathrm{y}$ \\
\hline StbMih7 & S. tuberosum & 6 & 0 & y & $\mathrm{y}$ & y \\
\hline StbMih8 & S. tuberosum & 6 & 0 & $y$ & $y$ & $y$ \\
\hline StbMih9 & S. tuberosum & 6 & 0 & $\mathrm{y}$ & $\mathrm{n}$ & $\mathrm{n}$ \\
\hline StbMih10 & S. tuberosum & 6 & 0 & $y$ & $\mathrm{n}$ & $\mathrm{n}$ \\
\hline StbMih11 & S. tuberosum & 6 & 0 & $\mathrm{y}$ & $\mathrm{n}$ & $\mathrm{n}$ \\
\hline StbMih12 & S. tuberosum & 6 & 0 & $y$ & $y$ & $y$ \\
\hline StbMih13 & S. tuberosum & 6 & 0 & $y$ & $\mathrm{n}$ & $\mathrm{n}$ \\
\hline StbMih14 & S. tuberosum & 6 & 0 & $y$ & $y$ & $y$ \\
\hline StbMih15 & S. tuberosum & 6 & 0 & $y$ & $\mathrm{n}$ & $\mathrm{n}$ \\
\hline StbMih16 & S. tuberosum & 6 & 0 & $\mathrm{y}$ & $\mathrm{n}$ & $\mathrm{n}$ \\
\hline StbMih17 & S. tuberosum & 6 & 0 & $\mathrm{y}$ & $\mathrm{y}$ & $\mathrm{y}$ \\
\hline StbMih18 & S. tuberosum & 6 & 0 & y & y & $y$ \\
\hline StbMih19 & S. tuberosum & 6 & 0 & $\mathrm{y}$ & $\mathrm{y}$ & $\mathrm{n}$ \\
\hline StbMih20 & S. tuberosum & 6 & 0 & $y$ & $\mathrm{n}$ & $\mathrm{n}$ \\
\hline StbMih21 & S. tuberosum & 6 & 0 & $y$ & $y$ & $\mathrm{n}$ \\
\hline StbMih22 & S. tuberosum & 6 & 0 & $\mathrm{y}$ & $\mathrm{y}$ & $\mathrm{n}$ \\
\hline StbMih23 & S. tuberosum & 6 & 0 & $\mathrm{y}$ & $\mathrm{y}$ & $\mathrm{n}$ \\
\hline StbMih24 & S. tuberosum & 6 & 0 & $\mathrm{y}$ & $\mathrm{n}$ & $\mathrm{n}$ \\
\hline StbMih25 & S. tuberosum & 6 & 0 & $\mathrm{y}$ & $\mathrm{y}$ & $\mathrm{n}$ \\
\hline StbMih26 & S. tuberosum & 6 & 0 & y & y & $\mathrm{n}$ \\
\hline
\end{tabular}




\begin{tabular}{|l|l|l|l|l|l|l|}
\hline StbMih27 & S. tuberosum & 6 & 0 & $\mathrm{y}$ & $\mathrm{n}$ & $\mathrm{n}$ \\
\hline StbMih28 & S. tuberosum & 6 & 0 & $\mathrm{y}$ & $\mathrm{n}$ & $\mathrm{n}$ \\
\hline StbMih29 & S. tuberosum & 6 & 0 & $\mathrm{y}$ & $\mathrm{y}$ & $\mathrm{n}$ \\
\hline StbMih30 & S. tuberosum & 6 & 0 & $\mathrm{y}$ & $\mathrm{y}$ & $\mathrm{n}$ \\
\hline StbMih31 & S. tuberosum & 6 & 0 & $\mathrm{y}$ & $\mathrm{n}$ & $\mathrm{n}$ \\
\hline StbMih32 & S. tuberosum & 6 & 0 & $\mathrm{y}$ & $\mathrm{n}$ & $\mathrm{n}$ \\
\hline StbMih33 & S. tuberosum & 6 & 0 & $\mathrm{y}$ & $\mathrm{n}$ & $\mathrm{n}$ \\
\hline StbMih34 & S. tuberosum & 9 & $2 . \mathrm{e}-111$ & $\mathrm{y}$ & $\mathrm{n}$ & $\mathrm{n}$ \\
\hline StbMih35 & S. tuberosum & 5 & $2 . \mathrm{e}-116$ & $\mathrm{y}$ & $\mathrm{n}$ & $\mathrm{n}$ \\
\hline StbMih36 & S. tuberosum & 5 & 0 & $\mathrm{y}$ & $\mathrm{n}$ & $\mathrm{n}$ \\
\hline StbMih37 & S. tuberosum & 5 & $1 . \mathrm{e}-138$ & $\mathrm{y}$ & $\mathrm{n}$ & $\mathrm{n}$ \\
\hline StbMih38 & S. tuberosum & 5 & 0 & $\mathrm{y}$ & $\mathrm{n}$ & $\mathrm{n}$ \\
\hline
\end{tabular}

${ }^{a}$ The $\%$ identity was calculated by comparing to Mi-1.2 gene. 


\begin{tabular}{|c|c|c|}
\hline Exon 3 length (bp) & Exon $3 \%$ Identity $^{a}$ & Exon 3 Location \\
\hline 1656 & 94 & 2968283-2969939 \\
\hline 1130 & 83 & $54023408-54024538$ \\
\hline 1667 & 76 & $63127792-63126125$ \\
\hline 1629 & 76 & $63139824-63141453$ \\
\hline 2269 & 76 & $55466079-55463810$ \\
\hline 1659 & 77 & 58332176-58333835 \\
\hline 1627 & 76 & $63417394-63419021$ \\
\hline 1900 & 75 & 59752284-59754184 \\
\hline 717 & 74 & 56645512-56644795 \\
\hline 3717 & 96 & 2746865-2750582 \\
\hline 3714 & 95 & 2358513-2354799 \\
\hline 3695 & 95 & $2703202-2706897$ \\
\hline 3714 & 94 & $2331182-2327468$ \\
\hline 3708 & 92 & $2722412-2726120$ \\
\hline 3399 & 96 & 2377597-2374198 \\
\hline 4588 & 90 & 2321581-2316994 \\
\hline 2166 & 76 & $51405914-51408080$ \\
\hline 3145 & 85 & $26212074-26215219$ \\
\hline 3755 & 88 & $4666589-4662834$ \\
\hline 3185 & 89 & $4712285-4709100$ \\
\hline 3751 & 86 & $2782962-2779211$ \\
\hline 3710 & 87 & $4595915-4592205$ \\
\hline 3063 & 88 & $4381959-4385022$ \\
\hline 3700 & 85 & $4784199-4780499$ \\
\hline 3444 & 84 & $21415084-21411640$ \\
\hline 3097 & 86 & 2774764-2771667 \\
\hline 2800 & 86 & $9765061-9762261$ \\
\hline 5077 & 88 & $4585349-4580272$ \\
\hline 2643 & 86 & $4742038-4739395$ \\
\hline 3092 & 89 & 2830194-2827102 \\
\hline 2795 & 85 & $9740609-9737814$ \\
\hline 3104 & 89 & $4736112-4733008$ \\
\hline 3668 & 92 & $4620521-4616853$ \\
\hline 3710 & 87 & $4812638-4808928$ \\
\hline 2346 & 88 & $4795389-4793043$ \\
\hline 2641 & 86 & $4758050-4755409$ \\
\hline 1811 & 86 & 2789861-2788050 \\
\hline 3533 & 89 & 2816538-2813005 \\
\hline 2107 & 91 & $4767375-4765268$ \\
\hline 1739 & 87 & $4637843-4636104$ \\
\hline 850 & 88 & $4643409-4642559$ \\
\hline 1982 & 85 & $4706085-4704103$ \\
\hline
\end{tabular}




\begin{tabular}{|c|c|c|}
\hline 1290 & 81 & $4800415-4799125$ \\
\hline 988 & 84 & $9768426-9767438$ \\
\hline 943 & 84 & $4615975-4615032$ \\
\hline 991 & 88 & $4745801-4744810$ \\
\hline 825 & 85 & $4764990-4764165$ \\
\hline 727 & 86 & $4680706-4679979$ \\
\hline 1556 & 80 & $4373369-4374925$ \\
\hline 1489 & 77 & $36614185-36615674$ \\
\hline 1743 & 76 & $52767845-52769588$ \\
\hline 1542 & 80 & $25586240-25584698$ \\
\hline 1551 & 78 & $58707965-58706414$ \\
\hline 1970 & 76 & $58715465-58713495$ \\
\hline
\end{tabular}


Table S3: Sw5 homologues in the genome of Capsicum annuum

\begin{tabular}{|c|c|c|c|c|}
\hline Gene ID & Chromosome & E-value & $\%$ Identity $^{a}$ & Length (bp) \\
\hline CA03g03770 & 3 & 0 & 77 & 3650 \\
\hline CA10g13570 & 10 & 0 & 81 & 1013 \\
\hline CA03g05100 & 3 & 0 & 76 & 1505 \\
\hline CA07g05750 & 7 & 0 & 81 & 810 \\
\hline CA03g03760 & 3 & $1 \mathrm{e}-166$ & 77 & 1074 \\
\hline CA03g05110 & 3 & 6 e-164 & 85 & 583 \\
\hline CA07g16420 & 7 & 0 & 79 & 2677 \\
\hline CA03g03840 & 3 & 4 e-180 & 82 & 797 \\
\hline CA03g03380 & 3 & 0 & 77 & 2220 \\
\hline CA03g01330 & 3 & 0 & 79 & 1960 \\
\hline CA03g03360 & 3 & 0 & 78 & 2085 \\
\hline CA03g03350 & 3 & 9 e- 63 & 77 & 459 \\
\hline CA03g03920 & 3 & $2 \mathrm{e}-108$ & 75 & 1013 \\
\hline CA03g03970 & 3 & 0 & 79 & 1537 \\
\hline CA03g04650 & 3 & 0 & 78 & 3098 \\
\hline CA03g03710 & 3 & 0 & 82 & 1571 \\
\hline CA03g04670 & 3 & $2 \mathrm{e}-134$ & 84 & 515 \\
\hline CA07g05760 & 7 & $2 \mathrm{e}-104$ & 80 & 549 \\
\hline CA03g10900 & 3 & $1 \mathrm{e}-120$ & 81 & 786 \\
\hline CA03g03730 & 3 & 0 & 83 & 2004 \\
\hline CA03g03790 & 3 & 0 & 83 & 1325 \\
\hline CA03g03940 & 3 & 0 & 78 & 1357 \\
\hline CA03g03800 & 3 & 0 & 82 & 3715 \\
\hline CA03g03930 & 3 & 8 e-168 & 84 & 611 \\
\hline CA03g10910 & 3 & $1 \mathrm{e}-81$ & 86 & 288 \\
\hline CA03g03890 & 3 & 1 e-95 & 88 & 595 \\
\hline
\end{tabular}

${ }^{a}$ The \% identity was calculated by comparing to $S w 5-b$ gene. 
Table S4: Mi-1 homologues in the genome of Capsicum annuum .

\begin{tabular}{|c|c|c|c|c|}
\hline Gene ID & Fhromosome & E-value & Exon 3\% Identity ${ }^{a}$ & Length (bp) \\
\hline CA06g05130 & 6 & 0 & 82 & 1184 \\
\hline CA06g05120 & 6 & 0 & 85 & 832 \\
\hline CA02g24750 & 2 & 0 & 82 & 3044 \\
\hline CA06g00820 & 6 & $2 \mathrm{e}-164$ & 87 & 516 \\
\hline CA06g03680 & 6 & 4 e-171 & 74 & 658 \\
\hline CA00g30030 & unknown & 0 & 83 & 3135 \\
\hline CA06g01250 & 6 & 0 & 82 & 2123 \\
\hline CA06g01210 & 6 & $1 \mathrm{e}-135$ & 84 & 526 \\
\hline CA06g02810 & 6 & 0 & 85 & 1008 \\
\hline CA06g01190 & 6 & 0 & 82 & 2322 \\
\hline CA00g80340 & unknown & 0 & 83 & 3117 \\
\hline CA06g00980 & 6 & 0 & 83 & 1712 \\
\hline CA06g02200 & 6 & 0 & 82 & 1280 \\
\hline CA06g02160 & 6 & 0 & 85 & 1009 \\
\hline CA06g02100 & 6 & 0 & 85 & 816 \\
\hline CA06g00950 & 6 & 0 & 84 & 778 \\
\hline CA06g03410 & 6 & 0 & 86 & 665 \\
\hline CA06g02040 & 6 & 5 e-95 & 86 & 338 \\
\hline CA06g02030 & 6 & 0 & 82 & 1261 \\
\hline CA06g02620 & 6 & $1 \mathrm{e}-76$ & 78 & 801 \\
\hline CA06g02520 & 6 & 0 & 84 & 1896 \\
\hline CA06g02680 & 6 & 0 & 84 & 834 \\
\hline CA06g05110 & 6 & 0 & 84 & 896 \\
\hline CA06g03630 & 6 & 0 & 80 & 2230 \\
\hline CA06g01040 & 6 & 6 e-119 & 79 & 639 \\
\hline CA06g02180 & 6 & 3 e-176 & 85 & 631 \\
\hline CA00g76980 & unknown & 0 & 82 & 3509 \\
\hline CA06g01770 & 6 & 0 & 82 & 2349 \\
\hline CA06g02830 & 6 & 0 & 84 & 2709 \\
\hline CA06g02740 & 6 & 0 & 83 & 2709 \\
\hline CA06g00660 & 6 & 0 & 84 & 1680 \\
\hline CA06g01020 & 6 & 0 & 82 & 1550 \\
\hline CA06g01230 & 6 & 0 & 83 & 2614 \\
\hline CA06g01750 & 6 & 0 & 86 & 736 \\
\hline CA06g03650 & 6 & 0 & 83 & 2061 \\
\hline CA00g30020 & unknown & 0 & 83 & 3093 \\
\hline CA06g03690 & 6 & 0 & 82 & 1234 \\
\hline CA06g87560 & 6 & 0 & 82 & 1809 \\
\hline CA05g15170 & 5 & 0 & 82 & 2161 \\
\hline CA06g01180 & 6 & 0 & 83 & 3067 \\
\hline CA06g01350 & 6 & 0 & 85 & 774 \\
\hline CA06g02450 & 6 & 7 e- 84 & 83 & 354 \\
\hline CA06g00910 & 6 & 2 e-109 & 83 & 441 \\
\hline CA06g00890 & 6 & 0 & 85 & 1368 \\
\hline CA06g01110 & 6 & 0 & 85 & 772 \\
\hline
\end{tabular}




\begin{tabular}{|c|c|c|c|c|}
\hline CA00g87620 & unknown & 0 & 84 & 953 \\
\hline CA06g01700 & 6 & $6 \mathrm{e}-109$ & 81 & 516 \\
\hline CA06g00990 & 6 & 0 & 82 & 3177 \\
\hline CA00g30010 & unknown & 0 & 82 & 2434 \\
\hline CA06g03420 & 6 & 0 & 83 & 1224 \\
\hline CA06g02070 & 6 & 0 & 83 & 1088 \\
\hline CA06g00530 & 6 & 0 & 86 & 1667 \\
\hline CA06g01130 & 6 & 0 & 81 & 1241 \\
\hline CA06g01220 & 6 & 8 e-118 & 83 & 472 \\
\hline CA06g02730 & 6 & 0 & 81 & 1247 \\
\hline CA06g00770 & 6 & 0 & 83 & 3108 \\
\hline CA06g02530 & 6 & 0 & 85 & 669 \\
\hline CA06g00920 & 6 & 0 & 81 & 1674 \\
\hline CA06g02570 & 6 & 0 & 83 & 1141 \\
\hline CA06g02420 & 6 & 0 & 83 & 2945 \\
\hline CA06g01740 & 6 & 0 & 81 & 1830 \\
\hline CA06g00650 & 6 & $2 \mathrm{e}-109$ & 86 & 365 \\
\hline CA06g00730 & 6 & 0 & 84 & 840 \\
\hline CA06g00750 & 6 & 0 & 85 & 851 \\
\hline CA06g02150 & 6 & 0 & 83 & 2240 \\
\hline CA06g00720 & 6 & 0 & 81 & 3661 \\
\hline CA06g03640 & 6 & 0 & 82 & 2147 \\
\hline CA06g00610 & 6 & 0 & 77 & 1923 \\
\hline CA06g00960 & 6 & 2 e-73 & 85 & 275 \\
\hline CA06g00580 & 6 & 0 & 84 & 3153 \\
\hline CA06g02170 & 6 & 0 & 78 & 1883 \\
\hline
\end{tabular}

a The \% identity was calculated by comparing to Mi-1.2 gene. 